\title{
AVALIAÇÃO DO CICLO DE DIÁLOGOS BRASIL - CABO VERDE COM USO DE CONFERÊNCIA AUDIOVISUAL EM INTERNET DE CONEXÃO LIMITADA
}

\author{
Cycle Assessment Dialogues Brazil - Cape Verde with the Use \\ of Audiovisual Conference in Limited Internet Connection \\ Edith Maria Cardoso Costa Pereira'; Angélica Baptista Silva; \\ João Aprigio Guerra de Almeida ${ }^{3}$; Carlos Eduardo da Silva Figueiredo ${ }^{4}$
}

\begin{abstract}
Resumo Objetivos: Avaliar se houve translação de conhecimento no experimento em tempo real entre Brasil e Cabo Verde com uso de software de conferência por internet, especialmente no que diz respeito ao dispositivo. Materiais e Métodos: Ausculta por meio de formulários sobre visão dos participantes quanto ao conjunto de ferramentas utilizadas e sobre os interesses de capacitação dos profissionais de saúde no país e no continente africano. Resultados: Profissionais de Cabo Verde conseguiram acompanhar a exposição em tempo real, vendo o conferencista no Brasil e os diapositivos. Todos acharam o conteúdo adequado, que houve clareza nas exposições e visualizaram o expositor de maneira satisfatória. Participantes destacaram temas relacionados à prevenção e gestão em saúde e sugeriram seminários nos mesmos moldes para os quatro países africanos de língua portuguesa. Conclusão: Conferências por internet são compatíveis com processos de educação permanente em saúde à distância para profissionais em áreas isoladas.
\end{abstract}

Palavras-chave: Telemedicina, Telessaúde, Educação Continuada, Comunicação em Saúde.

Abstract Aims: This study aims to investigate health's knowledge translation in real time experiment by internet conference software, especially concerning with the use of IT device. Materials and Methods: It was analyzed the participants' impressions on the set of tools used as well as their interests in training of health professionals in the African continent. Results: According to professionals in Cape Verde, the exhibition in real time was well succeeded in watching, speech and slides occurred in Brazil. In their opinion, the content was appropriated; there was clarity in the presentation and display of the exhibitor was satisfactory, with emphasis on issues related to prevention and health management. They suggested seminars in the same way for African Portuguese-speaking countries. Conclusion: Internet conferences are compatible with ongoing distance education processes to health professionals in remote areas.

Keywords: Telemedicine, Telehealth, Continuing Education, Health Communication

1. E-mail: edithpereira.nutri@gmail.com. Coordenadora do Banco de Leite Humano do Hospital Dr. Agostinho Neto - Praia - Mestre em Ciências Nutrição e Alimentação; 2. Pesquisadora do Instituto Nacional de Saúde da Mulher, da Criança e do Adolescente Fernandes Figueira/Fiocruz - Doutora em Saúde Pública; 3 . PCoordenador da Rede de Bancos de Leite Humano - Doutor em Saúde da Mulher e da Criança; Médico do Instituto Nacional de Saúde da Mulher, da Criança e do Adolescente Fernandes Figueira da Fiocruz - Mestre em Saúde Coletiva. Recebido em 31/03/2014. Aceito, após revisão, em 05/06/2014. 


\section{Introdução}

O continente africano sofre com a falta de mão de obra qualificada na área da assistência à saúde pública bem como lacunas no processo de educação permanente de profissionais de saúde locais.

A inclusão digital da população e o acesso às tecnologias da informação e comunicação na África também são inferior aos locais em que o índice de desenvolvimento humano - IDH é mais alto.

Na área da saúde da criança e da mulher, a mortalidade materno-infantil é alta e os Objetivos de Desenvolvimento do Milênio não foram atingidos. Para combater o problema da má formação e a escassez de recursos humanos na área da saúde, atividades que vem sendo empreendidas no continente africano desde 1980 são as reuniões à distância com o uso da rede digital.

A literatura registra diversas atividades de tele-educação como parte das ações dos serviços de telessaúde, que podem ser uma resposta a este problema ${ }^{1-3}$. Entretanto, revelam-se escassos os estudos que privilegiam a palavra dos profissionais de saúde do continente africano sobre o impacto destas ações ${ }^{4}$.

O artigo apresenta resultados da experiência de cooperação internacional sul-sul na tele-educação voltada para saúde entre Brasil e Cabo Verde no âmbito da Comunidade dos Países de Língua Portuguesa (CPLP). Em 2011, Cabo Verde, uma ilha de colonização portuguesa com 500 mil habitantes, destaca-se como um país com taxa de alfabetização de $82,8 \%$. Contudo, a mortalidade materna em cada 100 mil nascidos vivos está em 48,4 e a taxa de mortalidade infantil é de 23 a cada mil nascidos vivos'.

O Hospital Agostinho Neto - HAN, em Praia, abriga desde 2011 o primeiro Banco de Leite Humano na África, resultante da Cooperação Internacional entre Brasil e Cabo Verde. Há pelo menos seis anos (20082014) compõe a Rede Internacional de Bancos de Leite
Humano, coordenada pelo Instituto Nacional de Saúde da Mulher, da Criança e do Adolescente Fernandes Figueira da Fiocruz - IFF/FIOCRUZ.

Neste contexto, em 2011, foi realizado I Ciclo de Diálogos Brasil \& Cabo Verde sobre biossegurança e enfermagem com participação de cerca de quinze profissionais do HAN e de uma dezena de profissionais do IFF, utilizando ferramentas de telessaúde para troca de saberes à distância. $O$ projeto prevê expansão desta experiência no IFF para municípios da Amazônia e países de língua portuguesa na África.

Apresenta-se, no artigo, a percepção de profissionais de saúde de hospital de referência de Cabo Verde, Hospital Agostinho Neto, sobre interações por meio de software de conferência via internet e o impacto desta atividade em seu cotidiano na assistência à saúde. Outra questão abordada é a percepção do grupo sobre temas e atividades que poderiam ser realizadas em seu país e na África lusófona.

O objetivo foi avaliar se houve translação de conhecimento no experimento, especialmente no que diz respeito ao dispositivo - conferência por internet e a visão do grupo quanto aos interesses de capacitação e educação permanente dos profissionais de saúde no país e no continente africano.

\section{Materiais e Métodos}

Trata-se de estudo de natureza qualitativa de ausculta aos profissionais de saúde de Cabo Verde, ouvintes das sessões virtuais com base no modelo teórico da teoria ator-rede, TAR. O conceito fundamental de translação/tradução do conhecimento deriva da TAR de Latour e outros (1994).

Por essa linha, traduzir ou transladar significa deslocar objetivos, interesses, dispositivos e seres humanos. A pesquisa translacional em saúde é uma modalidade de pesquisa que tem por objetivo diminuir a distância entre a produção do conhecimento e seu efetivo uso na 
prática cotidiana das ações em saúde nos serviços por meio de intervenções inovadoras para a população 5 .

Já a telessaúde é toda atividade em rede e mediada por computação que promove a translação de conhecimento entre a pesquisa e os serviços de saúde ${ }^{6}$. Nesse sentido, o presente estudo é uma pesquisa avaliativa de atividade de telessaúde com uso de dispositivos eletrônicos de conferência por internet, envolvendo Brasil e Cabo Verde.

A avaliação utilizada no estudo é a de quarta geração ${ }^{7}$, em que predomina a abordagem relacional, marcada pela negociação e empoderamento dos atores envolvidos nos programas avaliados ${ }^{8}$.

Para ouvir os profissionais, a pesquisa seguiu as seguintes etapas:

\section{- Projeto piloto com ferramenta de baixo custo;}

- Formulário de perguntas abertas e fechadas para avaliação da experiência.

- Relatório qualitativo, contendo os resultados sistematizados e prospecções.

Sete participantes completaram o ciclo, participaram do encontro de avaliação, em que foi explicado o instrumento, e responderam o formulário. O questionário se dividiu em duas partes: uma sobre o desempenho do dispositivo quanto à simulação da presença síncrona e assíncrona, a segunda se referia a atividades de telessaúde no país e na África como um todo.

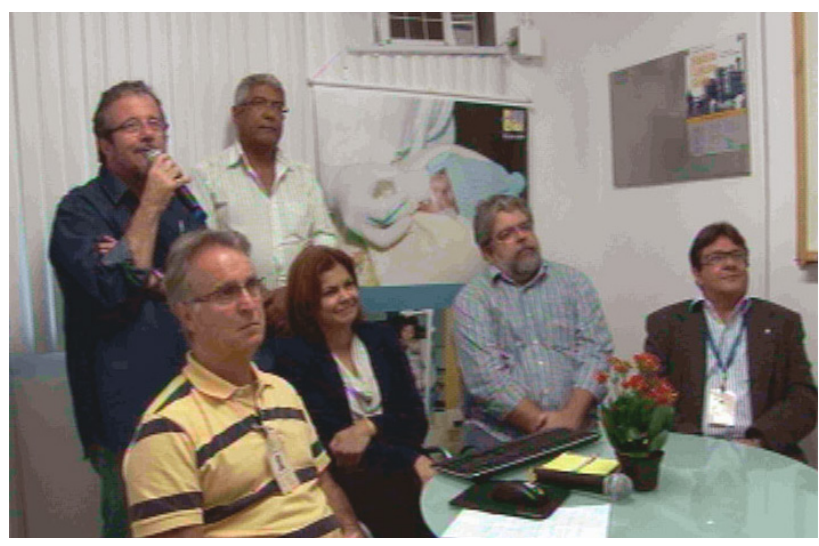

Figura 1. Pesquisadores no Rio de Janeiro interagem online com a equipe do Hospital Agostinho Neto em Cabo Verde.

\section{Resultados}

A experiência avaliada consistiu num ciclo de 10 encontros com duração de 3 horas em que profissionais do Instituto Nacional de Saúde da Mulher, da Criança e do Adolescente Fernandes Figueira apresentaram os seguintes temas: introdução a biossegurança; isolamento e lavagem das mãos; biossegurança e os bancos de leite humano; prematuridade e segurança alimentar; infecção do sítio cirúrgico (em dois momentos); infecção do trato urinário; infecção da corrente sanguínea; pneumonia associada à ventilação mecânica; imunização de profissionais de saúde.

O dispositivo que propiciou o encontro entre os profissionais dos dois hospitais foi a modalidade de software de conferência por internet, que simula uma sala em qualquer navegador. Ao entrar na sala, vários pontos podem habilitar áudio, vídeo, expor diapositivos, trocar

Figura 2.

Da esquerda para direita, visualização do dispositivo pelos participantes no RJ (Brasil) e em Praia (Cabo Verde).

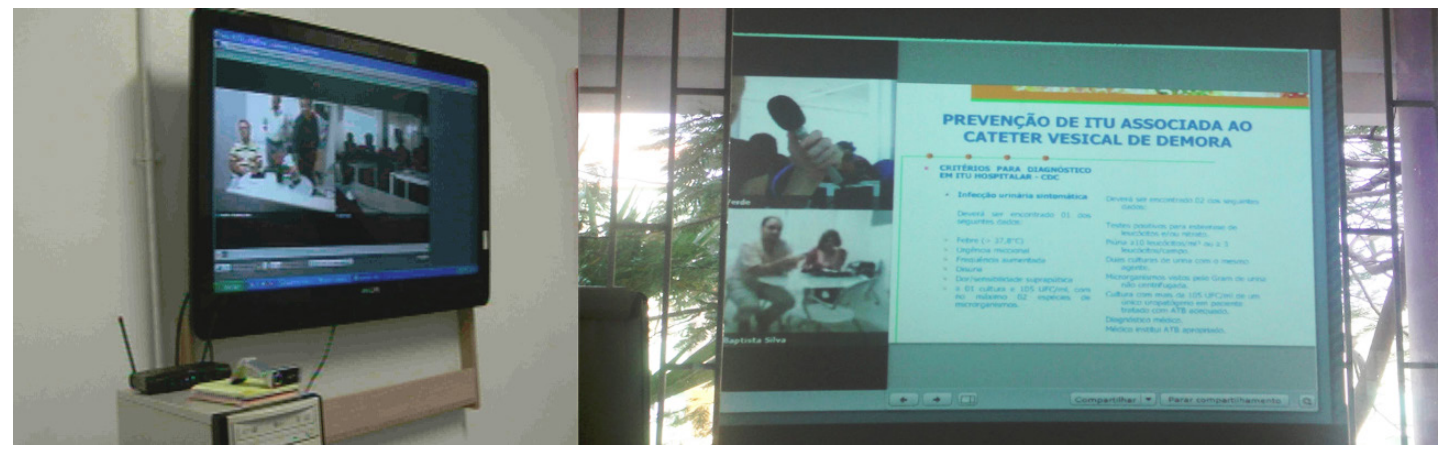


documentos e escrever em tempo real. A conexão utilizada foi a banda larga de internet comercializada nos dois países, sem qualquer tratamento de qualidade de serviço (QoS) específico para a atividade. Os profissionais se reuniram em um único ambiente e somente dois computadores (pontos) entravam na sala virtual. Em cada ambiente, no Rio de Janeiro (Brasil) e em Praia (Cabo Verde), havia um computador conectado a um telão e kit multimídia, que incluía microfone de mão.

Mesmo com problemas de conexão, os profissionais de Cabo Verde conseguiram acompanhar a exposição em tempo real, vendo o conferencista no Brasil e acompanhando os diapositivos. Todos acharam o conteúdo adequado, que houve clareza nas exposições e conseguiram ver o expositor de maneira satisfatória.

Os profissionais de saúde de Cabo Verde consideraram a conferência por internet com o uso de um navegador, projetada no telão e o computador ligado a caixas de som e microfones sem fio, uma ferramenta adequada para este tipo de atividade com uma exceção (Tabela1).

\begin{tabular}{|l|c|c|}
\hline QUESTÕES & SIM & NÃO \\
\hline Adequação do Conteúdo & 7 & - \\
\hline Clareza na Exposição & 7 & - \\
\hline Enquadramento do Expositor & 7 & - \\
\hline Ferramenta Adequada & 6 & 1 \\
\hline
\end{tabular}

Tabela 1. Opinião dos participantes quanto às conferências em tempo real.

\begin{tabular}{|l|c|c|}
\hline \multicolumn{3}{|c|}{ ESCALA 0-5 (grau crescente) } \\
\hline ALUNOS & ÁUDIO & VÍDEO \\
\hline Aluno 1 & 3 & 3 \\
\hline Aluno 2 & 4 & 5 \\
\hline Aluno 3 & 3 & 4 \\
\hline Aluno 4 & 2 & 2 \\
\hline Aluno 5 & 3 & 5 \\
\hline Aluno 6 & 3 & 4 \\
\hline Aluno 7 & 4 & 5 \\
\hline MÉDIA & 3,14 & 4 \\
\hline
\end{tabular}

Tabela 2. Grau de qualidade quanto à experiência audiovisual proporcionada pelo dispositivo.

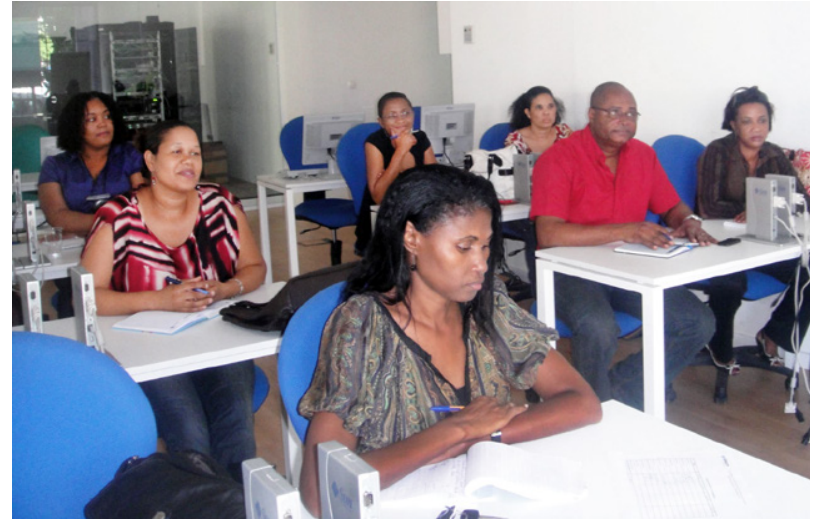

Figura 3. Em Cabo Verde, o grupo acompanha a fala e a apresentação no telão.

\begin{tabular}{|l|c|c|c|c|c|c|c|}
\hline \multicolumn{7}{|c|}{ MATERIAL ADICIONAL } \\
\hline ALUNOS & 1 & 2 & 3 & 4 & 5 & 6 & 7 \\
\hline Vídeos & 1 & - & - & - & 1 & 1 & 1 \\
\hline $\begin{array}{l}\text { Normas } \\
\text { Técnicas }\end{array}$ & - & - & 1 & 1 & - & - & 1 \\
\hline Áudio & - & - & - & & 1 & 1 & - \\
\hline Textos & - & - & - & 1 & 1 & - & - \\
\hline Livros & - & - & 1 & 1 & - & - & - \\
\hline Fotos & - & - & - & - & - & - & 1 \\
\hline
\end{tabular}

Tabela 3. Material hipermídia a ser utilizado nos encontros virtuais online.

O desempenho do vídeo por internet de baixa velocidade teve nota acima da média (Tabela 2). Porém, o resultado indica problemas com a compreensão do áudio.

Quanto à disponibilidade de material didático de maneia assíncrona, a maior demanda apontada pelo grupo foi de vídeos educativos, seguidos de normas técnicas (Tabela 3).

Indagados sobre temas e o tipo de telessaúde a ser desenvolvido no interior do país, o grupo sugeriu abordar 18 temas, sendo os mais apontados: cuidados com diabetes, doenças infectocontagiosas e gravidez precoce. No âmbito dos cuidados com a população adulta os temas sugeridos foram: obstetrícia; cuidados de enfermagem em ambiente hospitalar de maneira geral; gestão em enfermagem, cuidados com hipertensão e HIV; prevenção das doenças cardiovasculares, além da reciclagem em nível geral e nível específico de cuidados internos. 
Quanto à saúde infanto-juvenil, o grupo sugeriu: negligência com as crianças; cuidados na hospitalização; febre e diferença entre febre e hipertermia; cuidados com neoplasias, afecção gastrointestinal e respiratória, queimaduras, riastinia grave e sobre o procedimento atualizado na ingestão de soda cáustica.

Houve destaque no conjunto de respostas para temas relacionados à prevenção e gestão em saúde. Sobre a atividade em si o grupo destacou que seria interessante que outros profissionais tivessem oportunidade de se reciclarem na área de biossegurança sem ter que deslocar para outros países e com menos custo.

Um aluno afirmou que houve muitas informações e aprendizado. A iniciativa tem a vantagem de reunir grupos maiores, que precisam atualizar e aprofundar os seus conhecimentos. Essa experiência, segundo o grupo, permite atingir maior número de profissionais com baixo custo e formar profissionais capazes para melhorar a prestação de cuidados.

Quanto aos países africanos de língua portuguesa, os profissionais demonstraram muita preocupação com temas relacionados ao saneamento e chegaram a dizer que há necessidade de trabalhar todos os temas relacionados à saúde. Os assuntos a serem trabalhados na África lusófona na opinião da maioria dos profissionais foram a prevenção das doenças diarreicas e das doenças sexualmente transmissíveis. O cuidado com doenças cardiovasculares foi apontado de novo como tema, assim como as afecções gastrointestinais e respiratórias (superior e inferior) nas crianças. Na área materno-infantil destacam-se os cuidados com a negligência, vômito, vacinação, acompanhamento do pré-natal. Outros temas de abrangência geral foram biossegurança, saúde oral, higienização de água e alimentos.

Quanto à atividade, sugeriu-se que em nova oportunidade poderá ser feito seminário com representante dos quatro países - Cabo Verde, Angola, Moçambique, Guiné-Bissau, para intercâmbio de experiência na área de banco de leite. Segundo o grupo, cada país tem a sua experiência em termos epidemiológicos e essa poderia ser partilhada com todos de maneira a pensar como agirem sua realidade local.

Outra sugestão quanto à forma da atividade foi que houvesse intercâmbio entre países, reuniões formando grupos para educação permanente. A atividade, segundo os profissionais, deve englobar formações, apresentações de temas relacionados a problemas de saúde e educação com a realização de palestras.

\section{Discussão}

Tanto o continente africano quanto a região amazônica caracterizam-se por baixa inclusão digital e pouco acesso a redes de alta velocidade. Experiência ocorrida em 2012 no Brasil, que envolveu atividades de telessaúde e educação permanente, com a participação do Centro de Referência dos Bancos de Leite Humano e a Secretaria de Estado de Saúde do Amazonas, utilizou os mesmos dispositivos para um curso formal de capacitação com extremo êxito 9 .

Ambas as experiências demonstram que é possível organizar atividades de educação permanente em saúde em lugares remotos com conexão de internet instável e baixa com a vantagem de não tirar o profissional de seu lugar de trabalho.

A avaliação demonstra que é preciso escolher ferramentas adequadas e organizar a infraestrutura de acordo com a disponibilidade de banda de internet local. O primeiro passo é escutar as necessidades perfiladas pelos profissionais de saúde e a gestão dos sistemas de saúde participantes.

Dispositivos de conferência por internet têm interface adaptada a telefones celulares, o que torna este tipo de atividade mais acessível, pois a penetração de smartphones tem aumentado em todos os lugares do mundo, inclusive nas regiões com baixo $\mathrm{IDH}^{10}$. 
Além da formação recebida em biossegurança, a equipe do Banco de Leite de Cabo Verde foi capaz também de participar em sessões de apresentação e discussão de temas ligados ao Banco de Leite Humano na Rede Internacional.

Enfim, a translação do conhecimento em saúde através de processos de educação permanente com uso de telessaúde é uma necessidade para melhorar os serviços de saúde e diminuir as iniquidades, que a tecnologia da informação e da comunicação disponibiliza atualmente com baixo custo. Num País insular como é o caso de Cabo Verde e com fracos recursos econômicos, o recurso a tecnologias, como a telessaúde, é uma importante ferramenta de ensino e aprendizagem.

\section{Referências}

1. Kachieng'a MO. Challenges in managing diffusion of telemedicine technology in South Africa. Technology Management Conference (ITMC), 2011 IEEE International. 27 de junho de 2011;121-5.

2. Scott RE. Future proofing telehealth in developing countries. Journal of Telemedicine and Telecare. 2007;13(SUPPL. 3):70-2.

3. Dinis M, Santiago F, Silva L, Ferreira R, Machado J, Castela E. Telemedicine as a Tool for Europe-Africa Cooperation: A Practical Experience. In: Villafiorita A, Saint-Paul R, Zorer A, organizadores. E-Infrastructures and E-Services on Developing Countries [Internet]. Springer Berlin Heidelberg; 2010. p. 86-94. Disponívem em: http://dx.doi. org/10.1007/978-3-642-12701-4_10

4. Geissbuhler A, Bagayoko CO, Ly O. The RAFT network: 5 years of distance continuing medical education and tele-consultations over the Internet in French-speaking Africa. International Journal of Medical Informatics. maio de 2007;76(5-6):351-6.

5. Latour, B. Jamais fomos modernos: ensaio de antropologia simétrica. Tradução de Carlos Irineu da Costa. Rio de Janeiro: Editora 34, 1994. Coleção Trans.

6. Schmittdiel JA, Grumbach K, Selby JV. System-based participatory research in health care: an approach for sustainable translational research and quality improvement. Ann Fam Med. 2010;8(3):256-9. DOI:10.1370/afm.1117

7. Esher, Ângela et al. Construindo Critérios de Julgamento em Avaliação: especialistas e satisfação dos usuários com a dispensação do tratamento do HIV/Aids. Ciência \&amp; Saúde Coletiva, v. 17, p. 203-214, 2012.

8. Guba, E; Lincoln, Y. Fourth Generation Evaluation. Newbury Park: Sage, 1989.

9. Silva, AB; Silva, DA; Arreguy, EEM; Almeida,JAG. Curso de processamento e controle de qualidade do leite humano no Estado do Amazonas por telessaúde: um estudo de caso da Rede BLH do SUS. Revista Eletrônica de Comunicação, Informação \&amp; Inovação em Saúde; 7(2), 2013. DOI: 10.3395/reciis.v7i2.Sup1.725pt. Disponível em: http://www.reciis.icict.fiocruz.br/index.php/reciis/article/view/725/1626

10. Aranda-Jan CB, Mohutsiwa-Dibe N, Loukanova S. Systematic review on what works, what does not work and why of implementation of mobile health (mHealth) projects in Africa. BMC public health. 2014;14(1):188.int/trade/glossary/story021/en/ 